\title{
Emerging biomarkers in metastatic urothelial carcinoma: tumour mutational burden, PD-L1 expression and APOBEC polypeptide-like signature in a patient with complete response to anti-programmed cell death protein-1 inhibitor
}

\author{
Marcello Moro Queiroz ${ }^{1 \mathrm{a}}$ (iD, Zenaide Silva de Souza ${ }^{1 \mathrm{~b}}$ (iD, Aline Bobato Lara Gongora ${ }^{1 \mathrm{c}}$ (iD, Felipe de Galiza Barbosa ${ }^{2 \mathrm{~d}}$ (iD,

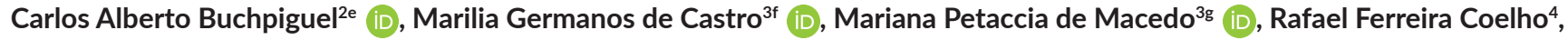 \\ Ethan Samuel Sokol ${ }^{5 h}$ (iD, Anamaria Aranha Camargo ${ }^{1 \mathrm{i}}$ (iD) and Diogo Assed Bastos ${ }^{1 \mathrm{j}}$ (iD) \\ ${ }^{1}$ Oncology Center, Hospital Sírio-Libanês (HSL), Rua Dona Adma Jafet, 91, São Paulo, SP, 01308-050, Brazil \\ ${ }^{2}$ Department of Diagnostic Imaging and Nuclear Medicine, Hospital Sírio-Libanês (HSL), Rua Dona Adma Jafet, 91, São Paulo, SP, 01308-050, Brazil \\ ${ }^{3}$ Department of Pathology, Hospital Sírio-Libanês (HSL), Rua Dona Adma Jafet, 91, São Paulo, SP, 01308-050, Brazil \\ ${ }^{4}$ Instituto do Câncer do Estado de São Paulo, Av Dr Arnaldo, 251 - Cerqueira César, São Paulo, SP, 01246-000, Brazil \\ ${ }^{5}$ Cancer Genomics Research, Foundation Medicine Inc., 150 Second St, Cambridge, MA 02141, USA \\ ahttps://orcid.org/0000-0001-5789-3397 \\ bhttps://orcid.org/0000-0002-9743-191X \\ chttps://orcid.org/0000-0002-2167-8166 \\ dhttps://orcid.org/0000-0002-3986-1778 \\ ehttps://orcid.org/0000-0003-0956-2790 \\ fhttps://orcid.org/0000-0001-8882-4650 \\ shttps://orcid.org/0000-0002-0434-7605 \\ hhttps://orcid.org/0000-0002-2988-0537 \\ ihttps://orcid.org/0000-0002-6076-9597 \\ ihttps://orcid.org/0000-0003-2480-353X
}

\begin{abstract}
Immunotherapy has recently been incorporated into the treatment guidelines for metastatic urothelial carcinoma. Nevertheless, the role of prognostic and predictive biomarkers in this setting is not completely defined. To date, PD-L1 expression and a high tumour mutational burden (TMB) seem to predict better responses to immune checkpoint inhibitors, but patients without these biomarkers may still respond to immunotherapy. There are some caveats regarding these biomarkers, such as lack of standardisation of techniques, tumour heterogeneity and other factors influencing the tumour microenvironment. Genomic signatures are other promising emerging strategies. We hereby discuss the management of a 70-year-old man with a metastatic recurrence of urothelial carcinoma within 1 year after neoadjuvant chemotherapy and radical cystectomy. Tumour next-generation sequencing showed a high TMB and a CD274 (PD-L1) amplification. The patient was treated with pembrolizumab and achieved a complete response.
\end{abstract}

Keywords: bladder cancer, metastatic, immunotherapy, biomarkers, tumour mutational burden, PD-L1
Correspondence to: Diogo Assed Bastos Email: diogo.bastos@hsl.org.br

ecancer 2021, 15:1306

https://doi.org/10.3332/ecancer.2021.1306

Published: $22 / 10 / 2021$

Received: $12 / 12 / 2020$

Publication costs for this article were supported by ecancer (UK Charity number 1176307).

Copyright: (c) the authors; licensee ecancermedicalscience. This is an Open Access article distributed under the terms of the Creative Commons Attribution License (http:// creativecommons.org/licenses/by/4.0), which permits unrestricted use, distribution, and reproduction in any medium, provided the original work is properly cited. 


\section{Introduction}

Bladder cancer is the fourth most common type of cancer in American men, with an estimate of 83.730 new cases and 34.130 deaths in the United States in 2021 [1]. Worldwide, there have been 549,000 new cases and 200,000 deaths due to bladder cancer in 2018 [2]. Cigarette smoking, occupational exposure to chlorine or arsenic and infectious agents are well established risk factors for this type of cancer. The exposure to certain drugs, such as cyclophosphamide and phenacetin-containing analgesics in high doses is related to the development of bladder cancer in humans [3].

Urothelial carcinoma is by far the most common histology of bladder cancer [4]. Muscle-invasive urothelial carcinoma is an aggressive disease, with a 5-year cancer-specific mortality of 37\%-67\% [5]. In metastatic bladder cancer, the scenario is even more discouraging: despite the high first-line response rates with platinum-based chemotherapy, the median overall survival (OS) varies between 11 and 16 months with conventional chemotherapy [6-9].

Over the last few years, cancer treatment is changing drastically with the development of new targeted therapies and immunotherapy. This paradigm-shift in cancer treatment includes urothelial cancer. As of September 2021, four immune checkpoint inhibitors (ICls) involving programmed cell death protein-1 (PD-1)/programmed death ligand-1 (PD-L1) pathway have been approved by Food and Drug Administration (FDA) for second-line treatment of metastatic urothelial cancer (mUC) [10]. More recently, avelumab, an anti-PD-L1, was approved for maintenance treatment for patients with no disease progression after first-line chemotherapy [11, 12] and Pembrolizumab/Atezolizumab were granted approval for first-line cisplatin ineligible patients [13]. Although these advances represent encouraging results, many efforts have been devoted towards discovering biomarkers that can be used in clinical practice as predictive markers of treatment response. Some of the molecular determinants that have been studied in other types of cancer and are currently being assessed in bladder cancer are PD-L1 expression, DNA mismatch-repair deficient (dMMR), DNA damage repair gene alterations [14], tumour mutational burden (TMB) and tumour-infiltrating lymphocytes (TILs) [15].

Here we reviewed the newest data about biomarkers of response to $\mathrm{ICl}$ and described a case report of a patient with metastatic bladder cancer whose tumour next-generation sequencing (NGS) showed a high TMB and a CD274 (PD-L1) amplification, and who presented a complete response (CR) after treatment with an $\mathrm{ICl}$, in order to illustrate this subject.

\section{Case report}

A 70-year-old man presented haematuria and a cystoscopy for investigation demonstrated a muscle-invasive urothelial carcinoma. A staging positron emission tomography/computed tomography with 2-deoxy-2[18-F]-fluoro-D-glucose (18-F FDG PET/CT) revealed an increased uptake on a parietal thickening of the right bladder wall with densification of the perivesical adipose tissue (standardised uptake values maximum (SUVmax): 12.9) and on right external iliac enlarged lymph nodes (LN), up to $3.0 \mathrm{~cm}$ (SUVmax: 20.1).

He then started on cisplatin-based chemotherapy with dense-dose methotrexate, vinblastine, doxorubicin and cisplatin (MVAC) for six cycles, presenting a partial response on imaging. After the last cycle, a radical cystoprostatectomy was performed and pathology showed a poor response to neoadjuvant chemotherapy (ypT3N2) (Figure 1). He was subsequently managed with close clinical and image follow-up.

Ten months after the cystoprostatectomy, an 18-F FDG PET/CT showed an increased uptake in mesorectal and inferior mesenteric LN (SUVmax: 7.9) and also in a nodule located on the right ischiatic foramen (SUVmax: 10.4). A biopsy of one of the suspicious LN confirmed metastatic urothelial carcinoma. The PD-L1 22C3 pharmDx immunohistochemistry (IHC) showed a Combined Positive Score (CPS) of 100. The paraffin-embedded tumour blocks from the LN metastasis were sent to FoundationOne CDX test. A NGS comprehensive panel was performed and showed a TMB of 18 muts/Mb, along with CD 274 (PD-L1) amplification, ERBB2 amplification, PTCH1 E1183*, CDKN1A R48*, JAK2 amplification, KDM6A V1113FS*7, MUTYH splice site 347-1G>C, PAX5 rearrangement exon 2, RB1 R787*, TERT promoter-146 C>T and TP53 R282W. Microsatellite status was stable and there were no FGFR alterations. An analysis of tumour mutation trinucleotide context revealed a dominant apolipoprotein B mRNA editing catalytic polypeptide-like (APOBEC) signature [16].

Due to the short time between exposure to MVAC chemotherapy and progression of disease, the poor previous response to platinum-based therapy and NGS results with biomarkers indicating a higher possibility of response to immunotherapy (high TMB and high PD-L1 expression), the patient was started on treatment with pembrolizumab.

An 18-F FDG PET/CT performed after the third cycle showed resolution of the glycolytic hypermetabolism in the mesorectal and inferior mesenteric $\mathrm{LN}$ and reduction in the right ischial foramen nodule. A new image after the seventh cycle demonstrated a CR (Figure 2), which 
was sustained after 9 months of treatment. The plan is maintenance of pembrolizumab until any limiting toxicity or disease progression. To the date of this publication, the patient remains with an ongoing CR with pembrolizumab.

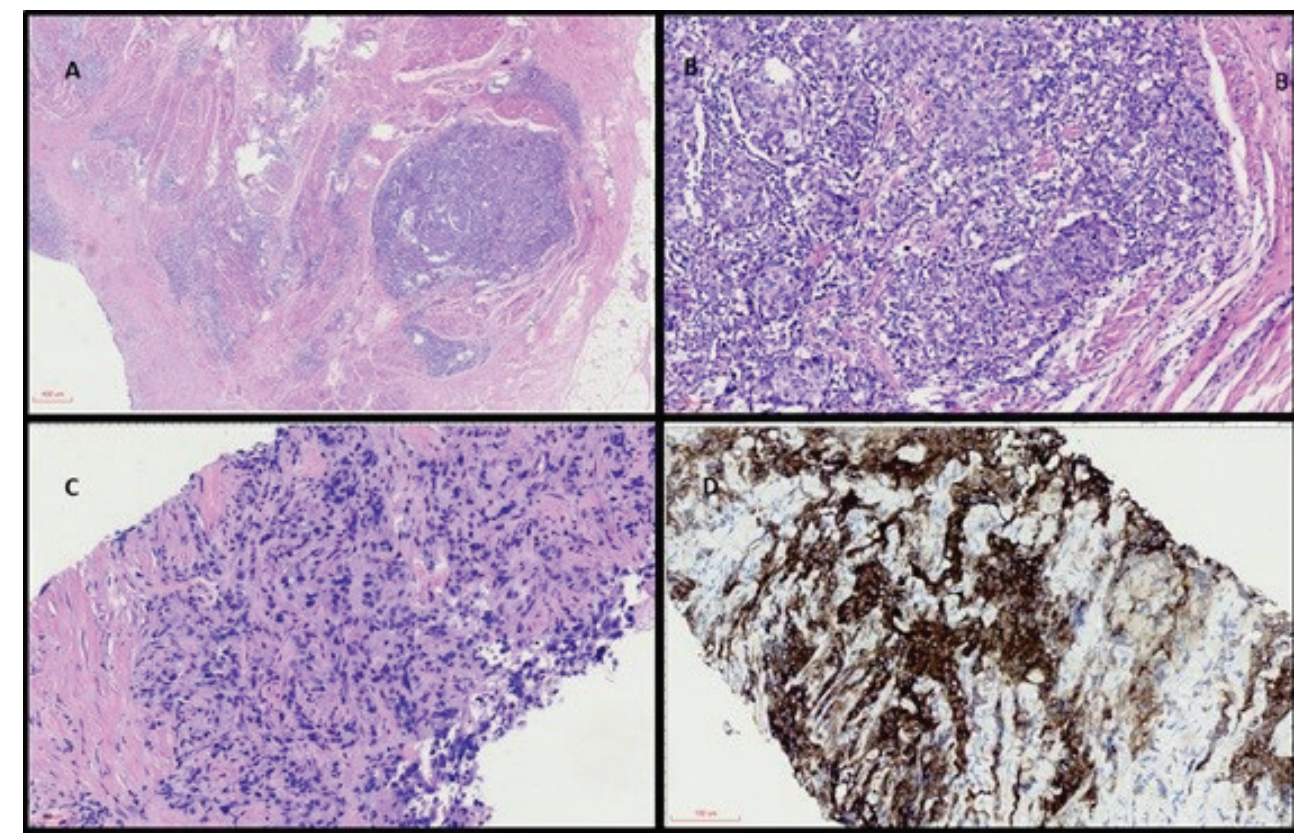

Figure 1 (coloured). (a): Panoramic view of haematoxylin and eosin (H\&E) stained sections of cystectomy specimen showing a highly infiltrative neoplasm through the vesical muscular wall (40x). (b): H\&E high power view analysis of cystectomy (200x) with invasive carcinoma in muscular propria and intratumoral lymphocyte infiltration. (c): H\&E analysis of metastatic lymph node with carcinoma (200x). (d): IHC with strong and diffuse positivity for PD-L1 22C3.

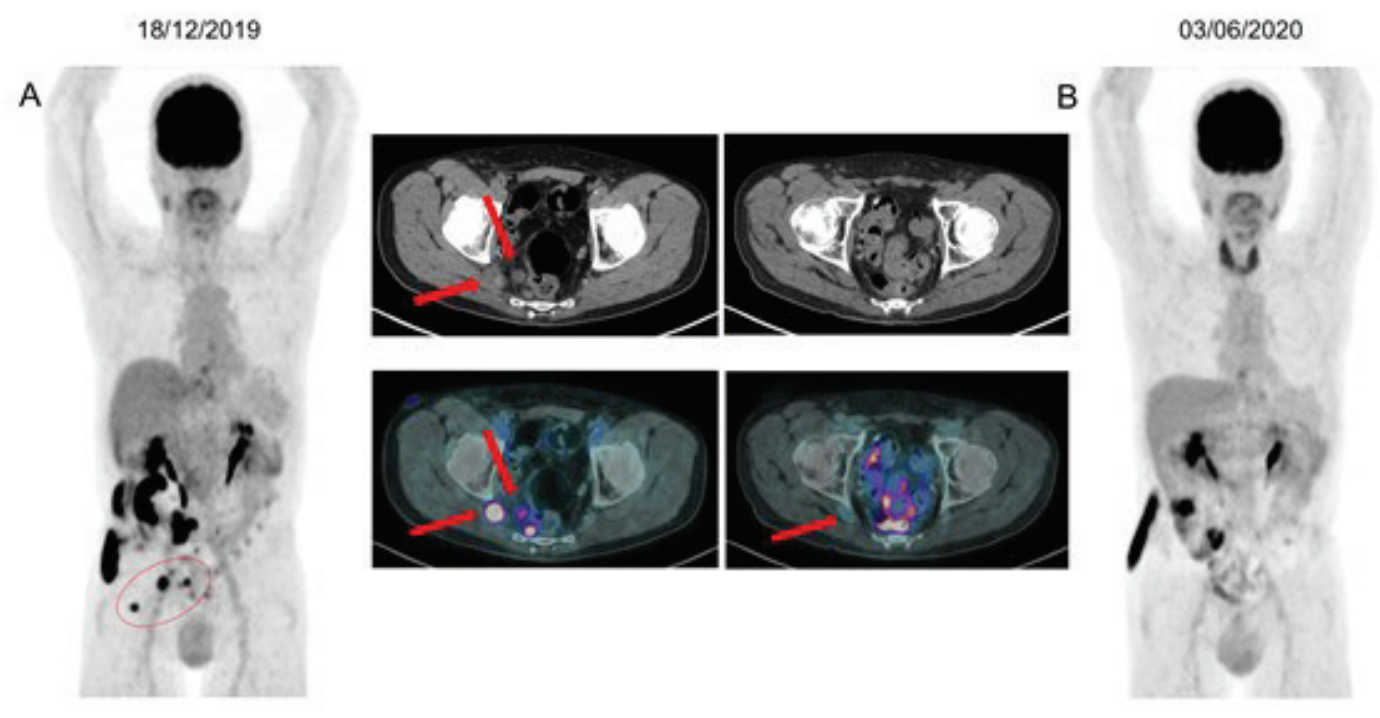

Figure 2. (a): 18F-FDG PET/CT imaging baseline before the initial therapy with pembrolizumab, showing mesorectal and inferior mesenteric LN, measuring up to $1.7 \mathrm{~cm}$ (SUVmax: 7.9). Nucleus centred on the right sciatic foramen measuring $2.0 \mathrm{~cm}$ (SUVmax: 10.4), maintaining close contact with the sciatic roots on this side. (b): Restaging after seventh cycle demonstrating resolution of glycolytic hypermetabolism in those previous LN. 


\section{Biomarkers of immunotherapy response in metastatic urothelial carcinoma}

Bladder cancer has a well-recognised course. Even when only non-muscle invasive bladder cancer is shown on the initial transurethral resection of bladder tumour, 50\%-70\% will develop multiple recurrences and $10 \%-20 \%$ will progress to muscle-invasive bladder cancer, conferring a worse prognosis [17].

Over the last 30 years, treatment regimens for mUC have remained almost unchanged [18, 19], favouring the use of platinum-based chemotherapies as first-line treatment, with no clear preferred option for second line [7]. However, with the development of PD-1/PD-L1 ICHs and targeted therapies, this paradigm has changed over the past few years [20].

In 2016, Atezolizumab was approved in second-line treatment of mUC by the FDA. This approval was based on the IMvigor 210 trial [10, 21]. Since then, four other anti-PD-1/PD-L1 therapies have been approved (Nivolumab, Durvalumab, Avelumab and Pembrolizumab) in the same scenario. Pembrolizumab is the only one with positive OS results in the second-line setting in a randomised phase III trial [22]. Most recently, atezolizumab and pembrolizumab were granted approval for first-line therapy in cisplatin-ineligible patients with overexpression of PD-L1. However, in June 2018, the FDA limited the use of both treatments in monotherapy for mUC based on a decreased survival when compared to platinum-based chemotherapy in patients who have not received prior therapy and have low expression of PD-L1. As a result, Pembrolizumab was indicated in cisplatin-ineligible patients and whose tumours express CPS $\geq 10$ or chemotherapy-ineligible patients, regardless of PD-L1 expression. For Atezolizumab, the indication was for cisplatin-ineligible patients with tumour infiltrating immune cells (IC) $\geq 5 \%$ of the tumour area and chemotherapy-ineligible patients regardless of PD-L1 [13, 23, 24]. Moreover, avelumab has shown significant benefit as maintenance therapy after not progressing to induction with first-line platinum-based chemotherapy, with improvement in OS and manageable side effects, and was recently FDA approved in this setting [11, 12].

These new therapies have demonstrated significant anti-tumour activity, with a median survival varying from 7 to 10.3 months, and an overall response rate (ORR) between $15 \%$ and $23 \%$ in the second-line setting (compared with the 10\% historical controls) [25]. In this same scenario, $5 \%$ and $7 \%$ of all patients achieved CR with Atezolizumab and Pembrolizumab, respectively, with a median time to response of 2.1 months with both $\mathrm{ICI}[22,26]$.

After the publication of trials evaluating the response to $\mathrm{ICI}$ in many mUC scenarios, and their following approval by the FDA, costeffectiveness analysis started to be made due to the high prices related to these new medications. For example, the cost-effectiveness of second-line Pembrolizumab was evaluated against chemotherapy or Atezolizumab in an American population, based on extrapolated results from KEYNOTE-045 and IMvigor-211. Results showed that despite the study limitations and at a $\$ 100.000$ willingness-to-pay threshold scenario, pembrolizumab and atezolizumab had a $66 \%$ and $100 \%$ probability of being cost-effective against chemotherapy, respectively [27]. Pembrolizumab was also evaluated against carboplatin-based chemotherapy in a PD-L1 positive first-line scenario using data from KEYNOTE-052. In this study, the use of the anti-PD-1 ICl resulted in a mean gain of 2.58 life years, 2.01 quality-adjusted life-years and additional costs of $\$ 158.561$, with a cost-effectiveness ratio of $\$ 78.925 /$ quality-adjusted life-year, when compared to carboplatin plus gemcitabine [28]. These analyses demonstrate that the use of this medication may be feasible, especially for patients that are likely to respond compared to other options (biomarker-driven strategy). The cost-effectiveness of pembrolizumab in second-line scenario was also suggested in the Swedish population; however, another study indicated that this medication can be cost-effective in the United States, but not in other countries [29, 30].

Nevertheless, concern has been raised on how to predict which patients would have better responses to ICl, since most patients unfortunately do not respond to this approach. In the pursuit of an answer to this question, many studies are evaluating possible biomarkers of response, with a special attention to PD-L1 expression evaluation by IHC and TMB [31, 32].

\section{PD-L1 expression by IHC}

PD-L1 expression evaluated by IHC in bladder cancer is associated with increased all-cause mortality and worse pathologic stage at resection, suggesting that high levels of PD-L1 expression may indicate more aggressive disease. The expression of this biomarker may be a 
prognostic factor and also predictive of response to PD-1/PD-L1 therapy [33, 34]. However, across clinical trials regarding mUC, PD-L1 assays and clinical results varied significantly, showing the challenge of using PD-L1 alone as a predictive biomarker. One explanation to this discrepancy is the lack of standardisation for PD-L1 detection between the studies. Of note, there are four distinct assays for PD-L1 IHC scoring used in the trials, with different scoring compartments for each specific therapy (some use tumour cell expression and others combine with immune cell expression) and distinct cut off values of PD-L1 expression (varying from 1\%, 5\%, 10\%, and 25\%). Moreover, PD-L1 as a tumour marker is dynamic over time and space, and a single biopsy may not be enough to represent the immune landscape in its entirety [25]. Most importantly, PD-L1 status is only a single factor in the tumour microenvironment. Other important features might more accurately segregate 'hot' from 'cold' tumours [35, 36].

\section{PD-L1 (CD274) amplification}

PD-L1 (CD274) amplification is relatively common in certain lymphomas and is associated with a high susceptibility to PD-1 blockade, but data are limited regarding solid tumours [37, 38]. In a retrospective study from Goodman et al [38], the prevalence of PD-L1 amplification in 118,187 solid tumours samples from the Foundation Medicine database was $0.7 \%$, and this alteration did not always correlate with highpositive PD-L1 expression by immunohistochemical analysis. In patients with PD-L1 amplified solid tumours that were treated with ICl, the objective response rate was 66.7\% (6/9), with a median progression-free survival of 15 months [38]. However, prospective studies with

larger number of patients are needed to confirm the correlation between PD-L1 amplification and responses to ICI, and there are no studies in urothelial cancer until now.

\section{Tumour mutational burden}

Regarding the TMB analysis, a variety of clinical studies have shown that patients with higher TMB experienced better prognosis, longer survival and greater response rates following treatment with $\mathrm{ICl}$ compared to those who have lower TMB levels [15, 39]. In patients with non-muscle invasive urothelial carcinoma treated with Bacillus of Calmette-Guérin immunotherapy, a high TMB was significantly associated with a higher response rate and recurrence-free survival [40]. Studies from melanoma and non-small cell lung cancer (NSCLC) suggest that the mutational load may potentially predict response more robustly than PD-L1 IHC, presence of TILs or clinical variables [41, 42]. When evaluating gene expression profiles and immune cell infiltration signatures in bladder cancer, Wu et al [43] indicated that TMB is closely related with immune microenvironment, suggesting that higher TMB tends to promote the infiltration of T cells and natural killer (NK) cells into the tumour microenvironment and thus, patients may achieve a more favourable prognosis. Beyond that, Wang et al [15] analysed the gene-set from The Cancer Genome Atlas (TCGA) database and correlated the expression of cancer-testis antigens (CTAs) with higher TMB patients. CTAs are a group of immunogenic proteins that are aberrantly activated in a variety of cancer types and are important targets for developing cancer immunotherapy.

Nevertheless, like PD-L1 expression, TMB has its limitations as a predictive biomarker. Many factors influence TMB assessment, which includes preanalytical factors, choice of the assay and methods of reporting (the last one can change depending on the assays and centres where the tests are done) [44]. The definition of what is considered a TMB high lacks standardisation between studies, as the cut-off value may vary depending on the publication, and the methods for calculating are inconsistent depending on the methodology used (for example, including or not synonymous mutations and Indels in the equation). Another problem is the technical limitation, depending on which NGS panel is used, because some of them may not include analysis of gene fusions, truncations and translocations, limiting the treatment evaluation when these alterations are present. Also, TMB is not constant during the whole treatment, meaning that its value may vary depending on which drugs the patient has been exposed to, thus demonstrating that a single biopsy during the whole treatment may not be sufficient to predict response to subsequent therapies. Lastly, germline variants may interfere with the results, as the techniques may not correctly filter common germline single nucleotide polymorphisms [25, 45].

Defects in DNA repair mechanisms, like microsatellite instability (MSI) and POLE (DNA polymerase epsilon) mutations, may work as surrogate measures for TMB and have emerged as potential biomarkers in the literature $[25,46]$. Patients in the metastatic scenario with MSI high and 
dMMR presented a better response rate when treated with pembrolizumab. Also, the phase II KEYNOTE 158 established the link between TMB-high status and improved ORR to anti-PD-1 in patients with various solid tumours. Based on these results and many others, the FDA granted Pembrolizumab agnostic approval for patients with solid tumours that carry the defects in dMMR, MSI high or TMB-high ( $\geq 10$ mutations/megabase) in the second-line scenario [47-50].

\section{Other biomarkers}

In an attempt to override the limitations in finding a reliable biomarker, there are numerous examples of studies across solid tumour types including head and neck squamous cell cancer, NSCLC, melanoma, and urothelial cancer exploring correlation between composite markers and response to anti-PD-1 [51, 52]. For example, in the subgroup analysis of the phase III IMvigor 130, a study in locally advanced or MUC, Atezolizumab monotherapy was related to improved OS in patients with PD-L1 in tumour infiltrating IC $\geq 5 \%$ (IC $2 / 3$ on the VENTANA SP142 assay) plus high TMB (10 mut/Mb cut-off), when compared to placebo plus platin/gemcitabine, with an interim OS hazard ratio of $0.22(95 \% \mathrm{Cl}, 0.08-0.63)[23]$.

Although PD-L1 and TMB are the most common biomarkers in many studies, some new strategies to predict response to ICl are being intensively investigated. Regarding the inflammatory status of the tumour, immune expression profiling is more accurate to determine a 'hot' tumour than PD-L1 expression alone. This technology, which quantifies gene expression from multiple cell types within a biopsy specimen, has shown a better correlation to chemokines, cytokines and cell surface proteins [35, 36]. For example, in the Checkmate-275 trial, there was a correlation between the presence of IFN- $\gamma$ (Interferon gamma) gene signature and better response to Nivolumab in mUC $(p=0.0003)$ [53]. More recently, some Next-Generation RNA expression technologies allowed immune profiling of more than 700 genes [25].

Transcriptome profiling to classify bladder cancer in many distinct groups is also a promising technique to better predict responses. Some of these molecular subtypes of urothelial cancer were correlated to anti-PD-1/PD-L1 response in exploratory analyses [54-56]. For example, in IMvigor 210, the luminal cluster II subtype was correlated to a better response to Atezolizumab in $\mathrm{mUC}$ (ORR $=34 \%, p=$ 0.0017). This response was characterised by transcriptional signatures associated with presence of activated T-effector cells [26,57]. On the other hand, Checkmate 275 showed discrepant results with better responses to Nivolumab in the Basal I subtype (ORR = 30\%; luminal cluster II ORR $=\sim 25 \%$ ) [53]. These results show the difficulty to use the molecular subtypes as biomarkers, especially across agents, mostly because the molecular subtype criteria differ in each study and the best tissue to be biopsied (primary tumour or metastatic lesions) is not standardised [25].

Another growing biomarker is the evaluation of the mutational signature associated with the APOBEC family enzymes, present in approximately $80 \%$ of bladder cancers [58]. Despite having a different physiologic function correlated to anti-viral defence in normal cells, in the tumour cells these enzymes are likely correlated with hypermutation at cytosine bases in exposed single-stranded DNA [59]. NGS analyses, such as the TCGA and others, have identified that this mutational signature is characterised by a TCW > T/C mutation [58, 60, 61]. Tumours can be divided into APOBEC-high and low tumours. The first ones are more likely to have mutations in DNA damage response genes (TP53, ATR, BRCA2) and chromatin regulatory genes (ARID1A, MLL, MLL3), potentially leading to a hypermutation phenotype and subsequent enhanced immune response against the tumour [58]. For instance, in the IMvigor-130 trial, mUC patients with APOBEC mutational signature had significantly higher TMB and improved OS with atezolizumab containing regimens in the first-line cisplatin ineligible scenario [23]. Despite this promising correlation, more trials are still needed to confirm APOBEC high mutational signature as a reliable biomarker of response to immunotherapy, especially with other ICls.

Table 1 summarises the main biomarkers in urothelial cancer and their features. 
Table 1. Biomarkers in metastatic urothelial carcinoma and their main features.

\begin{tabular}{|c|c|c|c|}
\hline Biomarkers & Method & Advantages & Disadvantages \\
\hline PD-L1 & $\begin{array}{l}\text { Expression } \\
\text { analysed by IHC }\end{array}$ & $\begin{array}{l}\text { Rapid and low cost } \\
\text { Widely applied in } \\
\text { clinical trials }\end{array}$ & $\begin{array}{l}\text { Distinct cut off values on each clinical study with different immunotherapies: varying } \\
\text { from } 1 \%, 5 \%, 10 \% \text { and } 25 \% \\
\text { Dynamic over time and space } \\
\text { Does not represent the entire tumour microenvironment }\end{array}$ \\
\hline $\begin{array}{l}\text { PD-L1 (CD 274) } \\
\text { amplification }\end{array}$ & NGS & $\begin{array}{l}\text { Patients treated with } \\
\mathrm{ICI} \text { showed improved } \\
\text { ORR and median } \\
\text { progression-free } \\
\text { survival }\end{array}$ & $\begin{array}{l}\text { Need evaluation in the bladder cancer scenario } \\
\text { Need prospective trials with a larger number of patients } \\
\text { Did not always correlate with immunohistochemical } \\
\text { High cost }\end{array}$ \\
\hline TMB & $\begin{array}{l}\text { Expression } \\
\text { analysed by } \\
\text { cancer-related } \\
\text { genes panels } \\
\text { (NGS) }\end{array}$ & $\begin{array}{l}\text { Predict response to } \\
\text { treatment based on } \\
\text { immunotherapy in } \\
\text { urothelial bladder } \\
\text { cancer } \\
\text { Related with the } \\
\text { infiltration of T cells, } \\
\text { NK cells and CTAs } \\
\text { into the tumour } \\
\text { microenvironment }\end{array}$ & $\begin{array}{l}\text { Lack of standardisation regarding the definition of high TMB } \\
\text { Some gene fusions, truncations and translocations may not be covered by NGS tests } \\
\text { TMB can change depending on prior treatment } \\
\text { Gene panel assays present differences in their methodology, the number of genes } \\
\text { and types of mutations included }\end{array}$ \\
\hline $\begin{array}{l}\text { Immune expression } \\
\text { profiling }\end{array}$ & $\begin{array}{l}\text { Quantifies RNA } \\
\text { from multiple } \\
\text { cell types with } \\
\text { next-generation } \\
\text { RNA expression } \\
\text { technologies }\end{array}$ & $\begin{array}{l}\text { More accurate to } \\
\text { determine 'hot' } \\
\text { tumours } \\
\text { Better correlation to } \\
\text { chemokines, cytokines } \\
\text { and cell surface } \\
\text { proteins } \\
\text { Next-generation } \\
\text { RNA expression } \\
\text { technologies with } \\
\text { immune profiling } \\
\text { greater than } 700 \\
\text { genes }\end{array}$ & $\begin{array}{l}\text { Needs results from phase III prospective studies there are still ongoing } \\
\text { Multiple gene panels currently available } \\
\text { High cost }\end{array}$ \\
\hline $\begin{array}{l}\text { Transcriptome } \\
\text { profiling }\end{array}$ & $\begin{array}{l}\text { Gene expression } \\
\text { profiling based } \\
\text { on TCGA } \\
\text { Research } \\
\text { Network }\end{array}$ & $\begin{array}{l}\text { Correlation between } \\
\text { responses to } \\
\text { immunotherapy and } \\
\text { some subtypes }\end{array}$ & $\begin{array}{l}\text { Lack of standardisation, especially because molecular subtype criteria differ in each } \\
\text { study } \\
\text { Lack of standardisation in which is the best tissue to be biopsied (primary tumour or } \\
\text { metastasis) } \\
\text { Small cohorts until today } \\
\text { Low negative predictive value (responses in all four subtypes) } \\
\text { Does not assess the tumour microenvironment }\end{array}$ \\
\hline
\end{tabular}


Table 1. Biomarkers in metastatic urothelial carcinoma and their main features. (Continued)

\begin{tabular}{|l|l|l|l|}
\hline Biomarkers & Method & Advantages & Disadvantages \\
\hline APOBEC & NGS & $\begin{array}{l}\text { Hypermutational } \\
\text { phenotype and } \\
\text { enhanced immune } \\
\text { response }\end{array}$ & $\begin{array}{l}\text { Benefit in OS with atezolizumab } \\
\text { Need for more prospective trials with other ICI } \\
\text { High cost }\end{array}$ \\
& & $\begin{array}{l}\text { APOBEC high } \\
\text { correlated with better } \\
\end{array}$ & \\
& & OS with some ICl & \\
\hline
\end{tabular}

CTAs, Cancer-testis antigens; IC, Immune cell; IHC, Immunohistochemistry; NGS, Next-generation sequencing; TMB, Tumour mutational burden

\section{Conclusion}

As immunotherapy and other therapies arise as new opportunities for the treatment of MUC, there is an urgent need to identify predictive biomarkers in order to choose the best treatment for each patient. Lack of standardisation, difficulty to reproduce the tests and cost effectiveness are some of the challenges currently faced (Table 1). A comprehensive approach to analysis, including PD-L1, TMB and MSI, plus potential biomarkers of resistance is important to identify patient likelihood of response. Additionally, new and composite biomarkers are needed to better guide treatment with these new therapies.

\section{List of abbreviations}

Anti-PD-1, Inhibitor programmed cell death protein-1; Anti-PD-L1, Inhibitor programmed death ligand-1; APOBEC, Apolipoprotein B mRNA editing catalytic polypeptide-like; CPS, Combined positive score; CTAs, Cancer-testis antigens; CR, Complete response; FDA, Food and Drug Administration; 18-F FDG PET/CT, Positron emission tomography/computer tomography with 2-deoxy-2[18-F]-fluoro-D-glucose; IC, immune cells; ICI, Immune checkpoint inhibitors; IHC, Immunohistochemistry; dMMR, Mismatch-repair deficient; MSI, Microsatellite instability; mUC, Metastatic urothelial cancer; Muts/Mb, Mutations per megabase; MVAC, Methotrexate, vinblastine, doxorubicin and cisplatin; NGS, Next-generation sequencing; NK cells, Natural killer cells; NSCLC, Non-small cell lung cancer; ORR, Overall response rate; OS, Overall survival; PD-1, Programmed cell death protein-1; PD-L1, Programmed death ligand-1; SUV max, Standardised uptake values maximum; TCGA, The Cancer Genome Atlas; TILs, Tumour-infiltrating lymphocytes; TMB, Tumour mutational burden.

\section{Clinical practice points}

- Immunotherapy has changed the paradigm of mUC treatment. There are five ICHs approved in first- and second-line of treatment

- We report a case of CR with pembrolizumab and a favourable molecular analysis that could be associated with this outstanding response

- To this day, there are not many reliable biomarkers that predict response to immunotherapy

- There is an urgent need to identify feasible and cost-effective predictive biomarkers of response to immunotherapy. 


\section{Conflicts of interest}

Dr Bastos has received research funding from Janssen, Astellas and Pfizer, as well as honoraria and consulting fees from Janssen, Astellas, MSD, Bayer, Roche and AstraZeneca. Dr Lara Gongora has received speaker fees from MSD, Amgen, Janssen, Astellas, MSD, Bayer and AstraZeneca. The remaining authors declare that they have no known competing financial interests or personal relationships that could have appeared to influence the work reported in this paper.

\section{Ethical approval}

Informed consent was obtained from the patient for publication of this case report and accompanying images.

\section{Authors' contributions}

Marcello Moro Queiroz: writing original draft, online drafting, data collection.

Zenaide Silva de Souza: writing original draft, online drafting, data collection.

Aline Bobato Lara Gongora: writing-reviewing, supervision, methodology.

Felipe de Galiza Barbosa: writing-reviewing, figures development.

Carlos Alberto Buchpiguel: writing-reviewing, figures development.

Marilia Germanos de Castro: writing-reviewing, figures development, immunohistochemical analysis.

Mariana Petaccia de Macedo: writing-reviewing, figures development, immunohistochemical analysis.

Rafael Ferreira Coelho: writing-reviewing, methodology.

Ethan Samuel Sokol: writing-reviewing.

Anamaria Aranha Camargo: writing-reviewing, supervision.

Diogo Assed Bastos: conceptualization, writing-reviewing and supervision.

\section{Acknowledgments}

The authors would like to thank the patient for allowing us to share his case details and Foundation Medicine for sharing information regarding the molecular analysis of the tumour specimen.

\section{Financial disclosure}

This study was funded by Hospital Sírio-Libanês, São Paulo, Brazil. 


\section{References}

1. American Cancer Society (2020) Cancer Facts \& Figures 2020 (Atlanta: American Cancer Society) https://www.cancer.org/research/cancer-facts-statistics/all-cancer-facts-figures/cancer-facts-figures-2020.html Date accessed: 7/07/20

2. Bray F, Ferlay J, and Soerjomataram I, et al (2018) Global cancer statistics 2018: GLOBOCAN estimates of incidence and mortality worldwide for 36 cancers in 185 countries CA: Cancer J Clin 68 394-424 https://doi.org/10.3322/caac.21492

3. Ferrís J, Berbel O, and Alonso-López J, et al (2013) Factores de riesgo ambientales no ocupacionales asociados al cáncer vesical Actas Urol Esp 37 579-586 https://doi.org/10.1016/j.acuro.2013.02.004

4. The American Cancer Society Medical and Editorial Content Team (2019) What is bladder cacer? https://www.cancer.org/cancer/bladder-cancer/about/what-is-bladder-cancer.html Date accessed: 7/07/20

5. Hanna N, Trinh QD, and Seisen T, et al (2018) Effectiveness of neoadjuvant chemotherapy for muscle-invasive bladder cancer in the current real world setting in the USA Eur Urol Oncol $183-90$ https://doi.org/10.1016/j.euo.2018.03.001

6. Saxman SB, Propert KJ, and Einhorn LH, et al (1997) Long-term follow-up of a phase III intergroup study of cisplatin alone or in combination with methotrexate, vinblastine, and doxorubicin in patients with metastatic urothelial carcinoma: a cooperative group study JCO 15 2564-2569 https://doi.org/10.1200/JCO.1997.15.7.2564

7. Fisher MD, Shenolikar R, and Miller PJ, et al (2018) Treatment patterns and outcomes in stage IV bladder cancer in a community oncology setting: 2008-2015 Clin Genitourin Cancer 16 e1171-e1179 https://doi.org/10.1016/j.clgc.2018.07.025

8. Cheeseman S, Thompson M, and Sopwith W, et al (2020) Current treatment and outcomes benchmark for locally advanced or metastatic urothelial cancer from a large UK-based single centre Front Oncol 10167 https://doi.org/10.3389/fonc.2020.00167 PMID: 32154169 PMCID: 7044411

9. Niegisch G, Gerullis H, and Lin SW, et al (2018) A real-world data study to evaluate treatment patterns, clinical characteristics and survival outcomes for first- and second-line treatment in locally advanced and metastatic urothelial cancer patients in Germany $\mathrm{J}$ Cancer 9 1337-1348 https://doi.org/10.7150/jca.23162 PMID: 29721042 PMCID: 5929077

10. Ning Y, Suzman D, and Maher VE, et al (2017) FDA approval summary: atezolizumab for the treatment of patients with progressive advanced urothelial carcinoma after platinum-containing chemotherapy Oncologist 22 743-749 https://doi.org/10.1634/theoncologist.2017-0087 PMID: 28424325 PMCID: 5469588

11. U.S. Food and Drug Administration FDA approves avelumab for urothelial carcinoma maintenance treatment 2020 https://www. fda.gov/drugs/drug-approvals-and-databases/fda-approves-avelumab-urothelial-carcinoma-maintenance-treatment Date accessed: $9 / 07 / 20$

12. Powles T, Park SH, and et al (2020) Maintenance avelumab + best supportive care (BSC) versus BSC alone after platinum-based firstline (1L) chemotherapy in advanced urothelial carcinoma (UC): JAVELIN Bladder 100 phase III interim analysis JCO 38 LBA1-LBA1 https://doi.org/10.1200/JCO.2020.38.18_suppl.LBA1

13. U.S. Food and Drug Administration FDA limits the use of Tecentriq and Keytruda for some urothelial cancer patients 2018 https:// www.fda.gov/drugs/resources-information-approved-drugs/fda-limits-use-tecentriq-and-keytruda-some-urothelial-cancer-patients Date accessed: 16/09/21

14. Teo MY, Seier K, and Ostrovnaya I, et al (2018) Alterations in DNA damage response and repair genes as potential marker of clinical benefit from PD-1/PD-L1 blockade in advanced urothelial cancers JCO 36 1685-1694 https://doi.org/10.1200/JCO.2017.75.7740

15. Wang $X$ and Li M (2019) Correlate tumor mutation burden with immune signatures in human cancers BMC Immunol 204 https://doi. org/10.1186/s12865-018-0285-5 PMID: 30634925 PMCID: 6329192 
16. Zehir A, Benayed R, and Shah RH, et al (2017) Mutational landscape of metastatic cancer revealed from prospective clinical sequencing of 10,000 patients Nat Med 2 703-713 https://doi.org/10.1038/nm.4333

17. Apolo AB, Vogelzang NJ, and Theodorescu D (2015) New and promising strategies in the management of bladder cancer Am Soc Clin Oncol Educ Book 105-112 https://doi.org/10.14694/EdBook_AM.2015.35.105 PMID: 25993148 PMCID: 6777550

18. Bellmunt J, Orsola A, and Leow JJ, et al (2014) Bladder cancer: ESMO practice guidelines for diagnosis, treatment and follow-up Ann Oncol 25 iii40-iii48 https://doi.org/10.1093/annonc/mdu223 PMID: 25096609

19. Milowsky MI, Rumble RB, and Booth CM, et al (2016) Guideline on muscle-invasive and metastatic bladder cancer (European Association of Urology Guideline): American Society of Clinical Oncology Clinical Practice Guideline Endorsement JCO 34 1945-1952 https:// doi.org/10.1200/JCO.2015.65.9797

20. Ghasemzadeh A, Bivalacqua TJ, and Hahn NM, et al (2016) New strategies in bladder cancer: a second coming for immunotherapy Clin Cancer Res 22 793-801 https://doi.org/10.1158/1078-0432.CCR-15-1135

21. Hoffman-Censits JH, Grivas P, and Van Der Heijden MS, et al (2016) IMvigor 210, a phase II trial of atezolizumab (MPDL3280A) in platinum-treated locally advanced or metastatic urothelial carcinoma (mUC) JCO 34 355-355 https://doi.org/10.1200/jco.2016.34.2 suppl.355

22. Bellmunt J, de Wit R, and Vaughn DJ, et al (2017) Pembrolizumab as second-line therapy for advanced urothelial carcinoma N Engl J Med 376 1015-1026 https://doi.org/10.1056/NEJMoa1613683 PMID: 28212060 PMCID: 5635424

23. Galsky MD, Arija JÁA, and Bamias A, et al (2020) Atezolizumab with or without chemotherapy in metastatic urothelial cancer (IMvigor130): a multicentre, randomised, placebo-controlled phase 3 trial Lancet 395 1547-157 https://doi.org/10.1016/S01406736(20)30230-0

24. Powles T, Csőszi T, and Özgüroğlu M, et al (2021) Pembrolizumab alone or combined with chemotherapy versus chemotherapy as first-line therapy for advanced urothelial carcinoma (KEYNOTE-361): a randomised, open-label, phase 3 trial Lancet Oncol 22 931-945 https://doi.org/10.1016/S1470-2045(21)00152-2 PMID: 34051178

25. Aggen DH, and Drake CG (2017) Biomarkers for immunotherapy in bladder cancer: a moving target J Immunotherapy Cancer 5 94 https://doi.org/10.1186/s40425-017-0299-1

26. Rosenberg JE, Hoffman-Censits J, and Powles T, et al (2016) Atezolizumab in patients with locally advanced and metastatic urothelial carcinoma who have progressed following treatment with platinum-based chemotherapy: a single-arm, multicentre, phase 2 trial Lancet 387 1909-1920 https://doi.org/10.1016/S0140-6736(16)00561-4 PMID: 26952546 PMCID: 5480242

27. Slater RL, Lai Y, and Zhong Y, et al (2020) The cost effectiveness of pembrolizumab versus chemotherapy or atezolizumab as secondline therapy for advanced urothelial carcinoma in the United States J Med Econ 23 967-977 https://doi.org/10.1080/13696998.2020. 1770261

28. Hale O, Patterson K, and Lai Y, et al (2021) Cost-effectiveness of pembrolizumab versus carboplatin-based chemotherapy as first-line treatment of PD-L1-positive locally advanced or metastatic urothelial carcinoma ineligible for cisplatin-based therapy in the United States Clin Genitourin Cancer 19 e17-e30 https://doi.org/10.1016/j.clgc.2020.07.006

29. Srivastava T, Prabhu VS, and Li H, Xu R, et al (2020) Cost-effectiveness of pembrolizumab as second-line therapy for the treatment of locally advanced or metastatic urothelial Carcinoma in Sweden Eur Urol Oncol 3 663-670 https://doi.org/10.1016/j.euo.2018.09.012

30. Sarfaty M, Hall PS, and Chan KKW, et al (2018) Cost-effectiveness of pembrolizumab in second-line advanced bladder cancer Eur Urol 74 57-62 https://doi.org/10.1016/j.eururo.2018.03.006

31. Kim JM and Chen DS (2016) Immune escape to PD-L1/PD-1 blockade: seven steps to success (or failure) Ann Oncol 27 1492-1504 https://doi.org/10.1093/annonc/mdw217 PMID: 27207108 
32. Lv J, Zhu Y, and Ji A, et al (2020) Mining TCGA database for tumor mutation burden and their clinical significance in bladder cancer Biosci Rep 40 BSR20194337 https://doi.org/10.1042/BSR20194337 PMID: 32239176 PMCID: 7178217

33. Boorjian SA, Sheinin Y, and Crispen PL, et al (2008) T-cell coregulatory molecule expression in urothelial cell carcinoma: clinicopathologic correlations and association with survival Clin Cancer Res 14 4800-4808 https://doi.org/10.1158/1078-0432.CCR-08-0731

34. Inman BA, Sebo TJ, and Frigola X, et al (2007) PD-L1 (B7-H1) expression by urothelial carcinoma of the bladder and BCG-induced granulomata: associations with localized stage progression Cancer 109 1499-1505 https://doi.org/10.1002/cncr.22588 PMID: 17340590

35. Gajewski TF (2015) The next hurdle in cancer immunotherapy: overcoming the non-T-cell-inflamed tumor microenvironment Semin Oncol 42 663-671 https://doi.org/10.1053/j.seminoncol.2015.05.011 PMID: 26320069 PMCID: 4555998

36. Sweis RF, Spranger S, and Bao R, et al (2016) Molecular drivers of the non-T-cell-inflamed tumor microenvironment in urothelial bladder cancer Cancer Immunol Res 4 563-568 https://doi.org/10.1158/2326-6066.CIR-15-0274 PMCID: 4943758

37. Miao D, Margolis CA, and Vokes NI, et al (2018) Genomic correlates of response to immune checkpoint blockade in microsatellitestable solid tumors Nat Genet 50 1271-12781 https://doi.org/10.1038/s41588-018-0200-2 PMCID: 6119118

38. Goodman AM, Piccioni D, and Kato S, et al (2018) Prevalence of PDL1 amplification and preliminary response to immune checkpoint blockade in solid tumors J Am Med Assoc Oncol 41237 https://doi.org/10.1001/jamaoncol.2018.1701

39. Klempner SJ, Fabrizio D, and Bane S, et al (2020) Tumor mutational burden as a predictive biomarker for response to immune checkpoint inhibitors: a review of current evidence Oncologist 25 https://doi.org/10.1634/theoncologist.2019-0244

40. Bastos DA, Mattedi RL, and Barreiro R, et al (2020) Genomic biomarkers and underlying mechanism of benefit from BCG immunotherapy in non-muscle invasive bladder cancer BLC 6 171-186 https://doi.org/10.3233/BLC-200289

41. Snyder A, Makarov V, and Merghoub T, et al (2014) Genetic basis for clinical response to CTLA-4 blockade in melanoma N Engl J Med 371 2189-2199 https://doi.org/10.1056/NEJMoa1406498

42. Rizvi NA, Hellmann MD, and Snyder A, et al (2015) Mutational landscape determines sensitivity to PD-1 blockade in non-small cell lung cancer Science 348 124-128 https://doi.org/10.1126/science.aaa1348

43. Wu Z, Wang M, and Liu Q, et al (2020) Identification of gene expression profiles and immune cell infiltration signatures between low and high tumor mutation burden groups in bladder cancer Int J Med Sci 17 89-96 https://doi.org/10.7150/ijms.39056 PMID: 31929742 PMCID: 6945555

44. Stenzinger A, Allen JD, and Maas J, et al (2019) Tumor mutational burden standardization initiatives: Recommendations for consistent tumor mutational burden assessment in clinical samples to guide immunotherapy treatment decisions Genes Chromosomes Cancer 58 578-588 https://doi.org/10.1002/gcc.22733 PMID: 30664300 PMCID: 6618007

45. Fernandez EM, Eng K, and Beg S, et al (2019) Cancer-specific thresholds adjust for whole exome sequencing-based tumor mutational burden distribution JCO Precision Oncol 1-12 https://doi.org/10.1200/PO.18.00400

46. Mehnert JM, Panda A, and Zhong H, et al (2016) Immune activation and response to pembrolizumab in POLE-mutant endometrial cancer J Clin Invest 126 2334-2340 https://doi.org/10.1172/JCI84940 PMID: 27159395 PMCID: 4887167

47. Diaz LA, Le DT, and Yoshino T, et al (2017) KEYNOTE-177: randomized phase III study of pembrolizumab versus investigator-choice chemotherapy for mismatch repair-deficient or microsatellite instability-high metastatic colorectal carcinoma JCO 35 TPS815-TPS815 https://doi.org/10.1200/JCO.2017.35.4_suppl.TPS815

48. Le DT, Uram JN, and Wang H, et al (2015) PD-1 blockade in tumors with mismatch-repair deficiency N Engl J Med 372 2509-2520 https://doi.org/10.1056/NEJMoa1500596 PMID: 26028255 PMCID: 4481136 
49. Marabelle A, Fakih MG, and Lopez J, et al (2019) Association of tumour mutational burden with outcomes in patients with select advanced solid tumours treated with pembrolizumab in KEYNOTE-158 Ann Oncol 30 v477-v478 https://doi.org/10.1093/annonc/ mdz253.018

50. U.S. Food and Drug Administration FDA approves pembrolizumab for adults and children with TMB-H solid tumors 2020 https://www. fda.gov/drugs/drug-approvals-and-databases/fda-approves-pembrolizumab-adults-and-children-tmb-h-solid-tumors Date accessed: $7 / 07 / 20$

51. Haddad RI, Seiwert TY, and Chow LQM, et al (2017) Genomic determinants of response to pembrolizumab in head and neck squamous cell carcinoma (HNSCC) JCO 35 6009-6009 https://doi.org/10.1200/JCO.2017.35.15_suppl.6009

52. Cristescu R, Mogg R, and Ayers M, et al (2017) Mutational load (ML) and T-cell-inflamed microenvironment as predictors of response to pembrolizumab JCO 35 1-1 https://doi.org/10.1200/JCO.2017.35.7_suppl.1

53. Sharma P, Retz M, and Siefker-Radtke A, et al (2017) Nivolumab in metastatic urothelial carcinoma after platinum therapy (CheckMate 275): a multicentre, single-arm, phase 2 trial Lancet Oncol 18 312-322 https://doi.org/10.1016/S1470-2045(17)30065-7

54. The Cancer Genome Atlas Research Network (2014) Comprehensive molecular characterization of urothelial bladder carcinoma Nature 507 315-322 https://doi.org/10.1038/nature12965 PMID: 24476821 PMCID: 3962515

55. Choi W, Porten S, and Kim S, et al (2014) Identification of distinct basal and luminal subtypes of muscle-invasive bladder cancer with different sensitivities to frontline chemotherapy Cancer Cell 25 152-165 https://doi.org/10.1016/j.ccr.2014.01.009

56. Damrauer JS, Hoadley KA, and Chism DD, et al (2014) Intrinsic subtypes of high-grade bladder cancer reflect the hallmarks of breast cancer biology Proc Natl Acad Sci 111 3110-3115 https://doi.org/10.1073/pnas.1318376111 PMCID: 3939870

57. Kojima T, Kawai K, and Miyazaki J, et al (2017) Biomarkers for precision medicine in bladder cancer Int J Clin Oncol 22 207-213 https:// doi.org/10.1007/s10147-016-1068-8

58. Glaser AP, Fantini D, and Wang Y, et al (2018) APOBEC-mediated mutagenesis in urothelial carcinoma is associated with improved survival, mutations in DNA damage response genes, and immune response Oncotarget 9 4537-4548 https://doi.org/10.18632/oncotarget.23344 PMCID: 5796993

59. Lada AG, Dhar A, and Boissy RJ, et al (2012) AID/APOBEC cytosine deaminase induces genome-wide kataegis Biol Direct 747 https:// doi.org/10.1186/1745-6150-7-47 PMID: 23249472 PMCID: 3542020

60. Lawrence MS, Stojanov P, and Polak P, et al (2013) Mutational heterogeneity in cancer and the search for new cancer-associated genes Nature 499 214-218 https://doi.org/10.1038/nature12213

61. Roberts SA, Lawrence MS, and Klimczak LJ, et al (2013) An APOBEC cytidine deaminase mutagenesis pattern is widespread in human cancers Nat Genet 45 970-976 https://doi.org/10.1038/ng.2702 